\title{
Review of Radiology Signs App for Android
}

\author{
Andy Wai Kan Yeung ${ }^{1}$ (i)
}

Published online: 20 July 2016

(C) Society for Imaging Informatics in Medicine 2016

\section{App Specs}

App Icon URL: https://h6.ggpht.com/SnldgbwPSFv1Sc9 Zg9N2AvUCFQQNwIxk4aVL7-auq3zbXaGuzsVfC8yrM6 QjcAMaJAU=w300-rw

App Name: Radiology Signs

App Developer: Androidmedics

App Developer Website: https://play.google. com/store/apps/developer?id=Androidmedics

App Price: free

Apple App Store URL: NA

Google Play Store URL: https://play.google.com/store/ apps/details?id=com.androidmedics.radiologysigns

Category: educational

Tags: clinical reference, educational, free, radiology, flashcard, Android-only

Works Offline: Yes

FDA Approval: NA

Promotion Code: None

\section{Quick Review}

(1 star: lowest / 5 stars: highest)

Overall Rating (1-5): 4

Content (1-5): 3

Usability (1-5): 4

Andy Wai Kan Yeung

ndyeung@hku.hk

Oral and Maxillofacial Radiology, Applied Oral Sciences, Faculty of Dentistry, The University of Hong Kong, 1B39B, Prince Philip Dental Hospital, 34 Hospital Road, Sai Ying Pun, Hong Kong
Pros: Comprehensive list of radiology signs. Can "bookmark" individual radiology signs of interest.

Cons: Sorted alphabetically only but not according to systems. Ad on top is distracting.

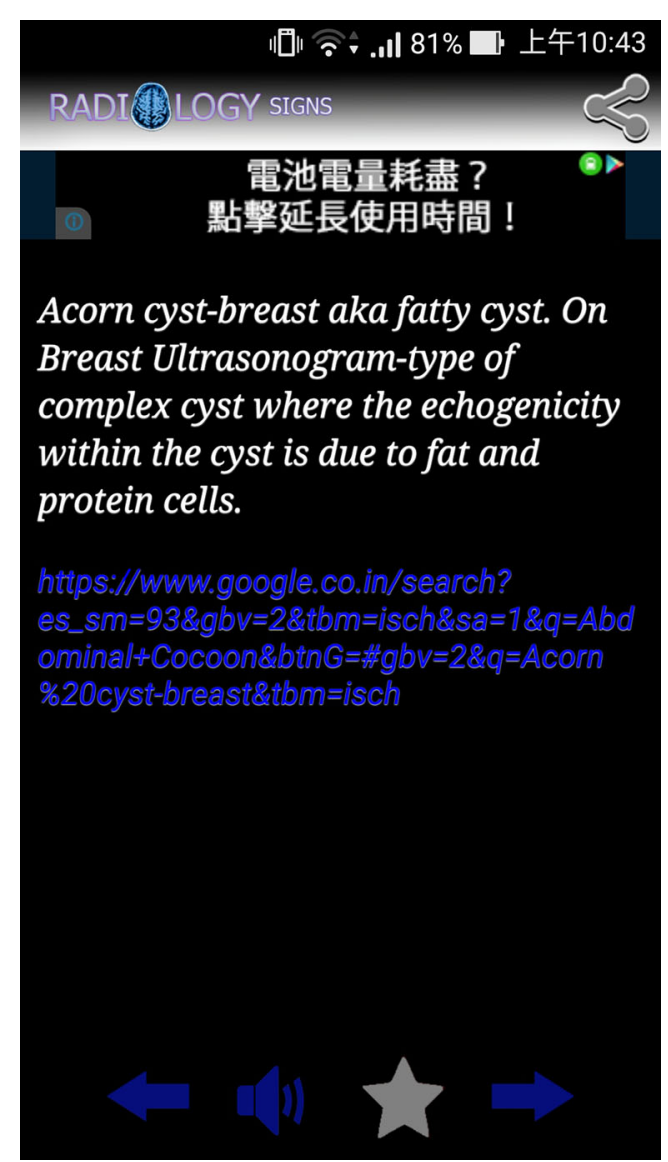

Fig. 1 One exemplary page of the app. Each entry consists of an explanatory text and a link to relevant images searched by Google 


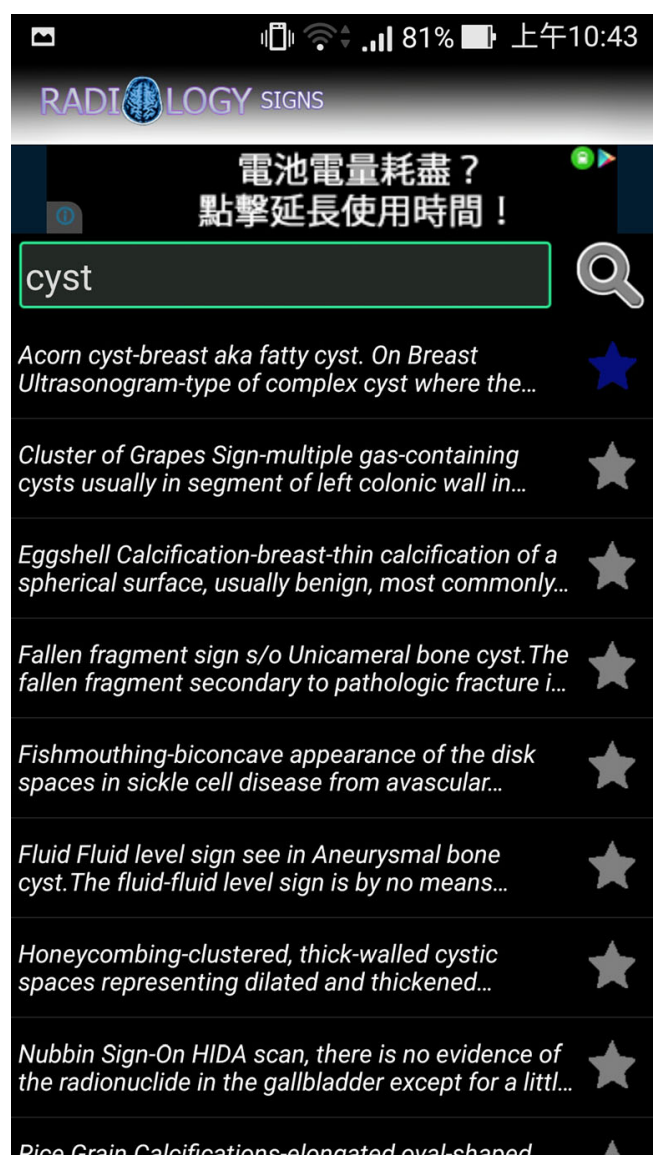

Fig. 2 Searching the list by keywords. There is no restriction on the position of the keywords in the explanatory text

At A Glance: A great go-to app for checking radiology signs, with short text and link to relevant images by Google search.

\section{Full Review}

\section{Intro}

There is a long list of common radiology signs involving various body systems from head to toe. Identifying the signs and recalling their clinical relevance are crucial to not only radiologists but also general practitioners with access to clinical images. This app provides a ready-to-use and convenient reference list for all healthcare workers equipped with Android mobile phones.

\section{Purpose/Features/Content}

The purpose of this app is to provide explanation and image links to a comprehensive list of common radiology signs

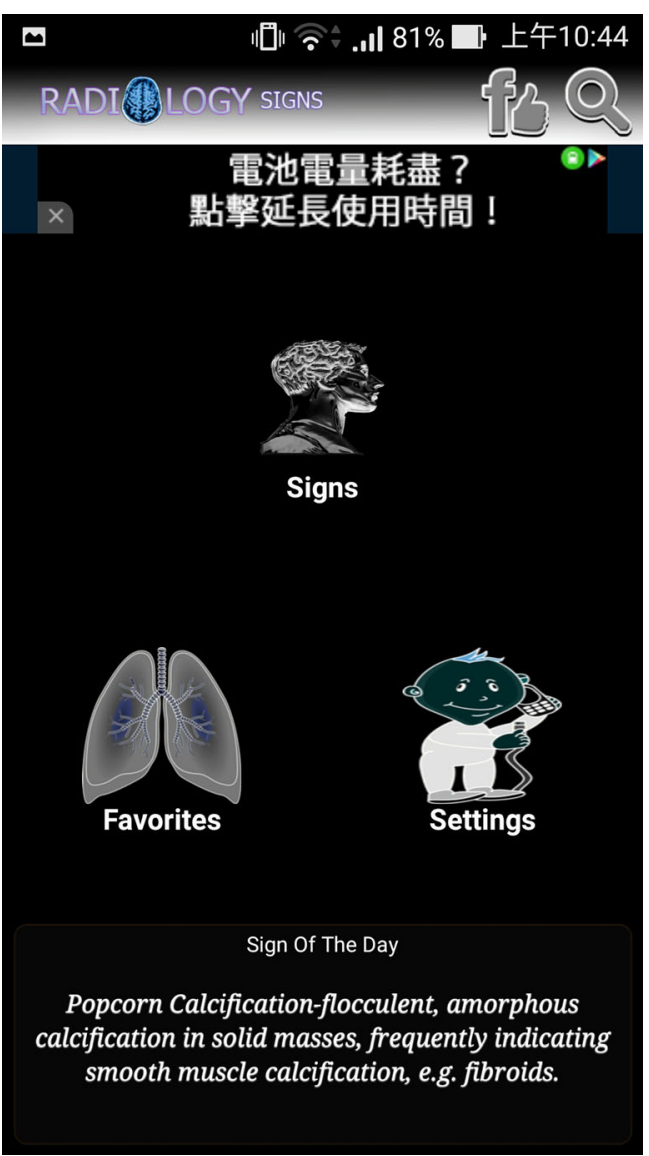

Fig. 3 The main page of the app. Bookmarked pages can be accessed by clicking the "Favorites" icon

observed in routine examinations ranging from x-ray, CT, MRI, US to NMR.

The whole list contains approximately 320 entries. Each entry consists of an explanation of the radiology sign and a link to relevant images searched with Google (Fig. 1).

There are two important features for accessing the list. The first is searching by entering words (Fig. 2). As long as the sign's name or its explanation contains the word, the search result will include it. The second is bookmarking favorite entries for easier re-visits by clicking the star beside the entries. A separate favorite list is retrieved with one-click access from the main page of the app (Fig. 3).

\section{Usability}

The app is straight-forward and easy to use. The layout is simple enough, and the black background makes the eyes comfortable even after prolonged reading. Font size 
and color are adjustable to suit individual preferences. Though the app does not have a landscape mode, its design well accommodates navigation by single hand in portrait mode.

\section{Good}

The app has clear explanation of the radiology signs and thus saves time from searching relevant information online. The page loading time is minimal, which allows direction to the exact explanatory text almost instantaneously.

\section{Room for Improvement}

There are four aspects to be improved. First, the text-tospeech "read out" function has a relatively robotic tone which is difficult to listen to. Second, the radiology signs are not sorted by system, e.g., cardiac, respiratory, etc. Third, the links to images are merely keyword searches with Google without validation of the accuracy of the images. Lastly, the advertisement placed at the top of the screen is sometimes quite distracting. 